Unraveling the impact of the Big Five personality traits on academic performance: the moderating and mediating effects of self-efficacy and academic motivation

Tim De Feyter Ralf Caers Claudia Vigna Dries Berings
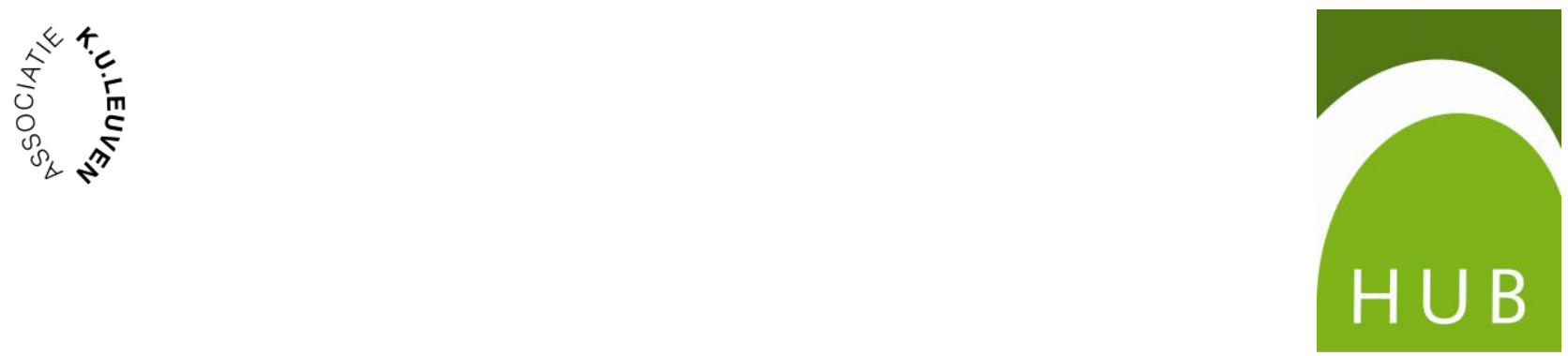


\title{
Unraveling the impact of the Big Five personality traits on academic performance: the moderating and mediating effects of self-efficacy and academic motivation
}

Tim De Feyter*, Ralf Caers, Claudia Vigna and Dries Berings

\begin{abstract}
The main purpose of this study is to unravel the impact of the Big Five personality factors on academic performance. We propose a theoretical model with conditional indirect effects of the Big Five personality factors on academic performance through their impact upon academic motivation. To clarify the mixed results of previous studies concerning the impact of neuroticism, we suggest a moderating role of self-efficacy. Hierarchical, moderated mediation and mediated moderation regression analyses were performed on longitudinal data collected from 375 students of a University college in Belgium. The findings revealed a positive indirect effect of neuroticism on academic performance at higher levels of selfefficacy, complemented by a positive direct effect of neuroticism at lower levels of selfefficacy. Finally, this study showed that conscientiousness positively affected academic performance indirectly through academic motivation, but also that it is a condition for the indirect impact of extraversion, neuroticism, and conscientiousness.
\end{abstract}

Keywords: Big Five personality traits, Self-efficacy, Academic motivation, Academic performance

* Corresponding author. E-mail: tim.defeyter@hubrussel.be 